\title{
Negative-Pressure Wound Therapy in a Patient with Osteomyelitis Caused by Multidrug-Resistant Bacterial Infection: A Case Report
}

\author{
Taekeun Yoon (D), Sang Wha Kim (D) \\ Department of Plastic and Reconstructive Surgery, Seoul National University Hospital, Seoul National University College of Medicine, Seoul, Korea
}

\begin{abstract}
Uncontrolled infection, especially with multidrug-resistant bacteria, may significantly inhibit wound healing. Although negative-pressure wound therapy can help prevent infection, it is contraindicated in patients with untreated infections, including osteomyelitis. Here, we report successful wound healing by negative-pressure wound therapy in a patient with refractory osteomyelitis due to multiple multidrug-resistant bacterial infections. Soft tissue infection and osteomyelitis progressed deeply through subcutaneous tunneling; hence, bacteria were not excreted from the affected area. Negative-pressure wound therapy reduced the bacterial load and resolved the patient's condition. Healing by secondary intention progressed with subsequent formation of granulation tissue. Despite recommendations against negativepressure wound therapy in patients with osteomyelitis, this study highlights the application of the technique for infection control in patients with multidrug-resistant bacterial infections and osteomyelitis.
\end{abstract}

Keywords: Fournier gangrene; Drug resistance, multiple, bacterial; Negative-pressure wound therapy; Osteomyelitis; Systemic inflammatory response syndrome

\section{Introduction}

Uncontrolled infection, especially with multidrug-resistant (MDR) bacteria, may inhibit wound healing. Although negative-pressure wound therapy (NPWT) may be useful in preventing infection by reducing bacterial bioburden [1,2], it is contraindicated in patients with untreated infections, including osteomyelitis [3-5]. Here, we describe successful wound healing through NPWT in a patient with refractory osteomyelitis caused by MDR bacterial infection. The soft tissue infection progressed deeply through subcutaneous tunneling from the right greater trochanter to the presacral area; hence, bacteria were not excreted from the affected area. In a severe case wherein no antibiotic was effective, dramatic wound healing was enabled by creating a small bone tunnel in the osteomyelitis lesion and applying NPWT to promote bacterial excretion. Despite several debates about whether NPWT can reduce bacterial load, in this case, NPWT application played a significant role in reducing bacterial bioburden in refractory infection. The study was approved by the Institutional Review Board of Seoul National University Hospital (IRB No. H-2109-106-1255) and performed in accordance with the principles of the Declaration of Helsinki. The patient provided written informed consent for the publication and the use of his images.

Case Report

Received: August 6, 2021

Revised: September 21, 2021

Accepted: September 22, 2021

Corresponding author: Sang Wha Kim, M.D., Ph.D.

Department of Plastic and Reconstructive Surgery, Seoul National University Hospital, Seoul National University College of Medicine, 101 Daehak-ro, Jongno-gu, Seoul 03080, Korea

Tel: $+82-2-2072-2375$

Fax: +82-2-741-3985

E-mail:sw1215@snu.ac.kr

This is an Open Access article distributed under the terms of the Creative Commons Attribution Non-Commercial License (https://creativecommons.org/licenses/by-nc/4.0/) which permits unrestricted non-commercial use, distribution, and reproduction in any medium, provided the original work is properly cited.

(c) 2021 Korean Wound Management Society 


\section{Case}

A 60-year-old man with a history of rectal cancer visited the emergency room for discomfort in the lower extremities and perineum persistently worsening for 2 months. Computed tomography showed abscesses in the pelvic cavity, presacral area, pelvic floor, right buttock, and thigh (Fig. 1), consistent with the diagnosis of Fournier gangrene. After several fasciotomies with debridement, above-knee amputation, and hospitalization for more than 2 months, the patient was referred to our department for soft tissue defect reconstruction. However, the infection was not controlled; thus, the patient was kept hospitalized. Reconstruction using rotational flaps was performed after 16 weeks of hospitalization, and split-thickness skin graft (STSG) was conducted after 17 weeks of hospitaliza- tion. Unfortunately, the infection resulted in loss of STSG, also affecting the rotational flaps.

Physical examination showed extensive soft tissue lesions around the pelvis, exposing the right greater trochanter (Fig. 2). Bacterial culture with antibiotic susceptibility tests showed infection with methicillin-resistant Staphylococcus aureus, carbapenem-resistant Acinetobacter baumannii, carbapenemresistant Pseudomonas aeruginosa, and vancomycin-resistant Enterococcus in the exposed soft tissue (Fig. 3). A magnetic resonance imaging scan was attempted to confirm osteomyelitis of the right pelvis; however, the imaging failed because the patient did not cooperate in the examination room. Subsequent treatment with antibiotics did not improve his condition. The patient later developed systemic inflammatory response syndrome (SIRS), which required administration of
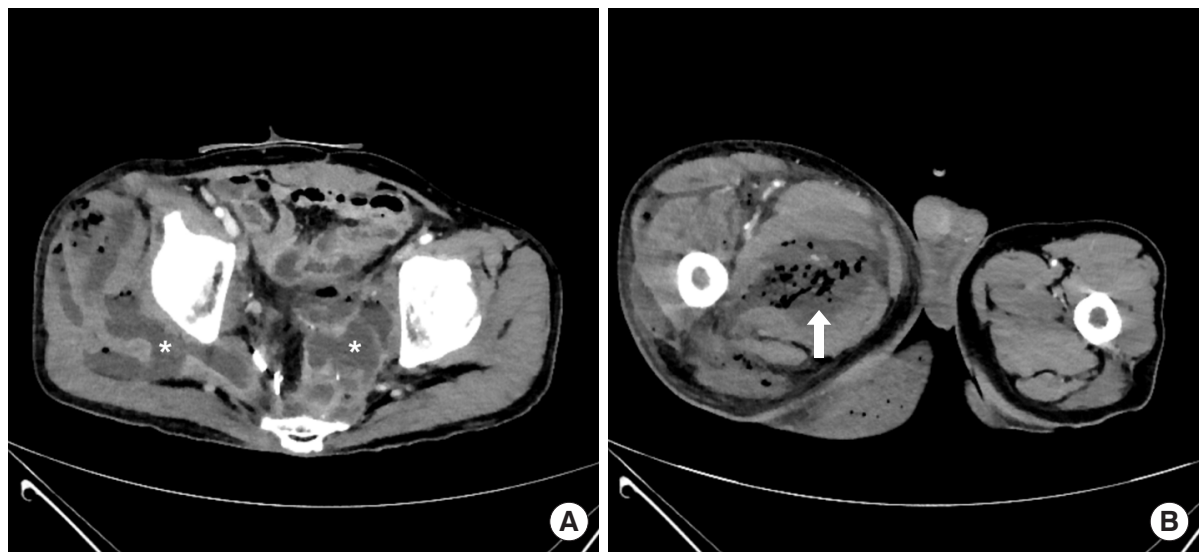

Fig. 1. Preoperative computed tomography findings. (A) Huge abscesses (asterisks) are visible in the pelvic cavity, presacral area, and pelvic floor. (B) The abscesses extend into the right buttock and thigh (arrow).
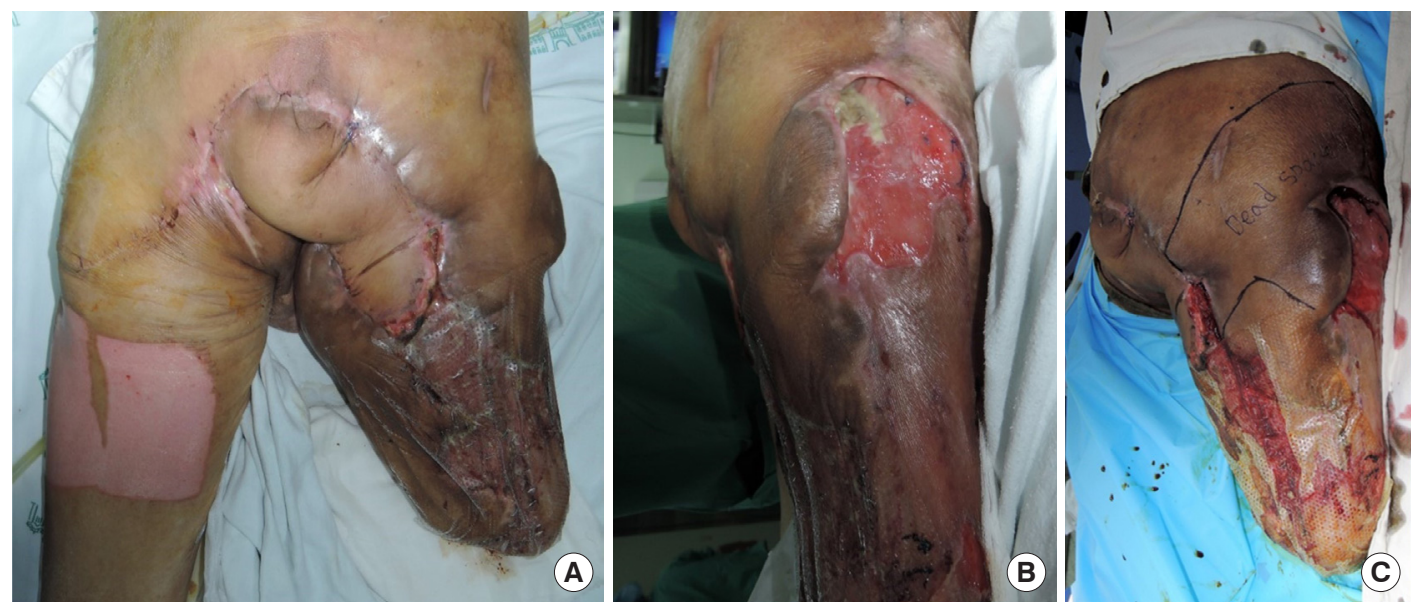

Fig. 2. Physical examination findings before negative-pressure wound therapy. (A, B) Progression of the soft tissue infection around the pelvis exposes the right greater trochanter. (C) Extensive soft tissue defects are observed as the uncontrolled infection in the dead space below the right buttock progresses. 


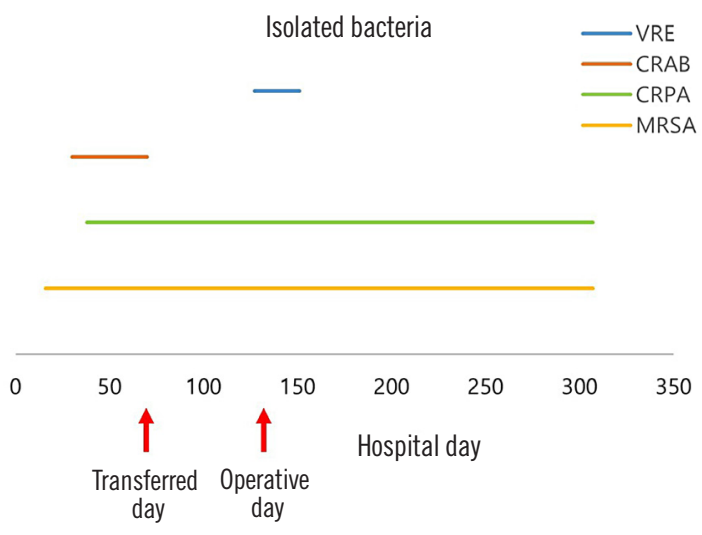

Fig. 3. Serial aspect of bacterial culture test after hospitalization. The serial aspect of multidrug-resistant bacterial culture test after hospitalization is shown. MRSA was identified first and detected last, and CRPA was similarly identified for a long period of time. MRSA, methicillin-resistant Staphylococcus aureus; CRAB, carbapenem-resistant Acinetobacter baumannii; CRPA, carbapenem-resistant Pseudomonas aeruginosa; VRE, vancomycin-resistant Enterococcus.

multiple antibiotics, including vancomycin. Nevertheless, the MDR bacterial infection was not controlled. Wound dressing was applied daily in the operating room to reduce bacterial burden. However, despite treatment, no improvement was observed in the lesion while fever and SIRS progressed. Infectious disease specialists recommended surgical resection of the extensive soft tissue lesions around the right pelvis, which was not feasible. With continued use of antibiotics, the patient's estimated glomerular filtration rate decreased from 79.8 to $37.7 \mathrm{~mL} / \mathrm{min} / 1.73 \mathrm{~m}^{2}$. The dead space from the right greater trochanter to the presacral area connected through subcutaneous tunneling was the suspected focus of infection; hence, it was difficult to sufficiently reduce the bacterial load by irrigation alone. Therefore, NPWT was considered.

To control infection, debridement of the right greater trochanteric area with creation of a small bone tunnel and marginal resection around the right pelvic bone were performed. NPWT was subsequently conducted on the affected areas to absorb the exudates from the dead space and reduce the bacterial burden. Osteomyelitis was confirmed from bone culture obtained in the operating room, and carbapenem-resistant Pseudomonas aeruginosa was identified. Immediately after treatment, a dramatic decrease in body temperature and C-reactive protein was observed (Fig. 4). Moreover, the patient's SIRS was resolved. NPWT dressings with standard polyurethane reticulated open-cell foam not containing silver were replaced every 3 days. Healing by secondary intention pro-
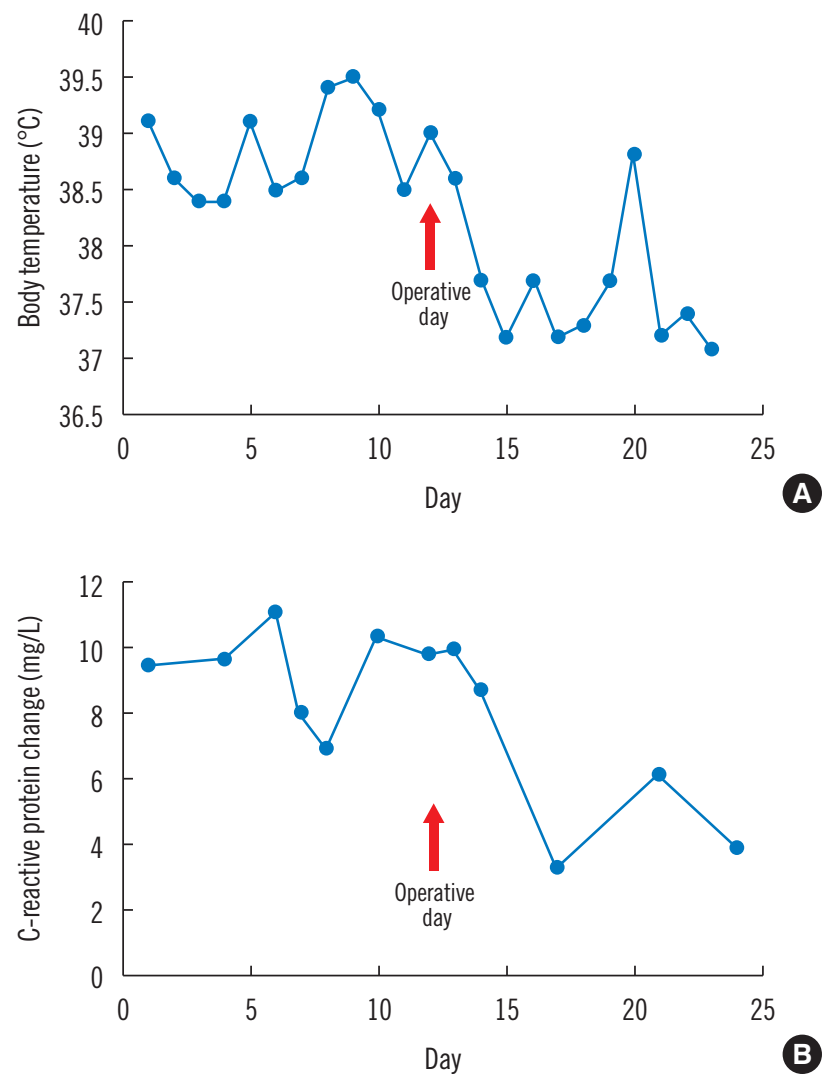

Fig. 4. Body temperature and C-reactive protein changes following negative-pressure wound therapy. (A) The graph shows the maximum body temperature per hospitalization day. A dramatic decrease in body temperature is observed immediately following negative-pressure wound therapy. (B) The graph shows the Creactive protein per hospitalization day. Additionally, C-reactive protein levels decreased after applying negative-pressure wound therapy.

gressed thanks to infection control and systemic improvement. Granulation tissue began to fill the clean wound area. NPWT was applied for 3 months and an STSG was used to successfully reconstruct the residual soft tissue defects (Fig. 5). To the best of our knowledge, NPWT for this variety of MDR bacterial infection has been rarely reported.

\section{Discussion}

Several studies have demonstrated the efficacy of NPWT in wound healing. NPWT promotes wound healing by inducing macro- and microdeformations, draining exudates, and creating an optimal environment for healing [6]. The representative mechanism of action of NPWT is to promote granulation tissue formation, increase blood flow to adjacent tissues, and remove bacteria from the wound bed $[1,2]$. Bacterial bioburden 

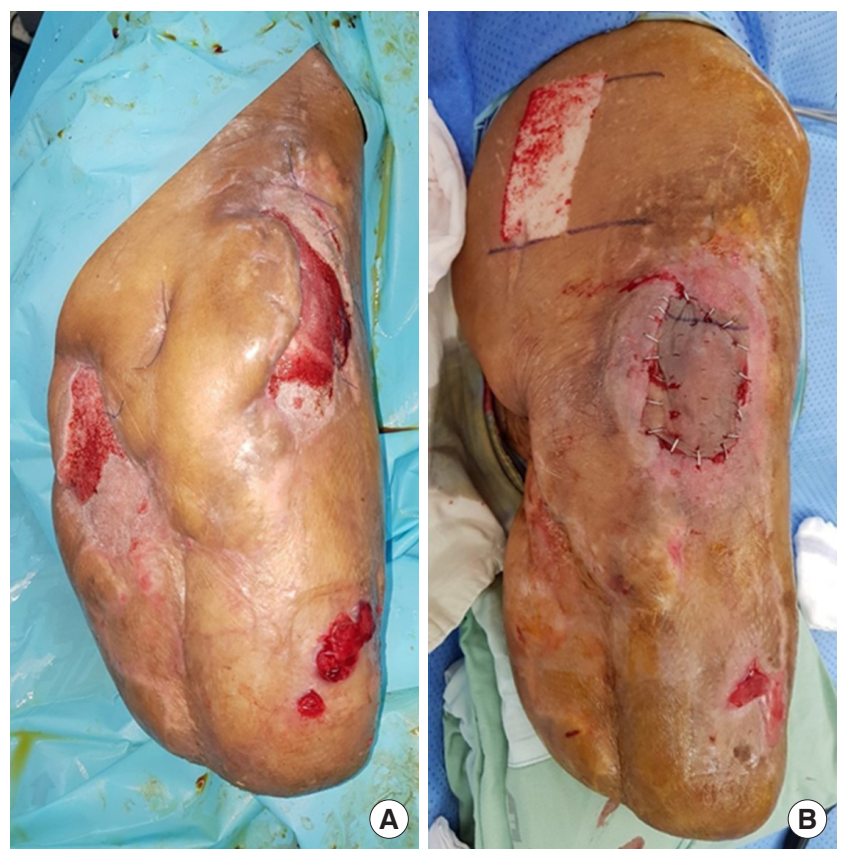

Fig. 5. Physical examination findings after negative-pressure wound therapy. (A) Negative-pressure wound therapy hastens wound healing and reduces the area of soft tissue defects. (B) The residual lesion is successfully reconstructed using a splitthickness skin graft.

increases metabolic demand; stimulates the proinflammatory environment; attracts monocytes, macrophages, and leukocytes that can interfere with wound healing; and secretes cytokines that cause vasoconstriction [7]. Therefore, reducing bacterial bioburden is crucial for wound healing, wherein application of NPWT can be helpful. According to a recent study, NPWT modulates cytokines to an anti-inflammatory profile and promotes mechanoreceptor- and chemoreceptor-mediated cell signaling by stress and hypoxia, leading to angiogenesis, extracellular matrix remodeling, and granulation tissue deposition [8]. In addition, proinflammatory cytokines altered by NPWT can trigger neutrophil accumulation, thereby accelerating bacterial clearance [9]. Nevertheless, whether NPWT reduces bacterial bioburden remains controversial.

Considering the various applications of NPWT, studies on the effect on bacterial bioburden are limited. In a descriptive analysis of the effect of conventional NPWT on bacterial load, eight of 20 studies revealed no effect on bacterial load, whereas seven demonstrated bacteriostatic activity and five showed species-selective inhibition of non-fermentative Gram-negative bacilli including Pseudomonas species [2]. In another study, quantitative cultures using a swab technique revealed that NPWT did not induce bacterial clearance in acute or chronic wounds but rather increased bacterial colonization, and nonetheless promoted wound healing [10]. This shows the complexity of wound healing that cannot be explained by a single factor. In another study, after applying NPWT to chronic wounds, sonication fluid of the NPWT foam was cultured. As wound healing progressed, the bacterial load did not decrease and even new bacteria were identified in some cases [11]. Since a certain number of bacteria are present in the foam of NPWT, bacterial load may increase in situations such as the foam not being replaced frequently. However, NPWT drains exudate from the wound, and in cases where the removed bacterial contents are of greater volume then the amount accumulated in the foam, bacterial bioburden may be reduced.

In the present case, bacterial infection was within the wound, making it difficult to treat. NPWT can eliminate and, at the same time, aggravate bacterial infection; therefore, reducing the bacterial load in the dead space was more crucial. Depending on the wound, the benefits of NPWT may be significant, even for infected lesions [12]. A randomized control study showed that NPWT shortened the healing period of infected wounds [13]. The choice to consider NPWT in controlling infection should be based on anatomic involvement and patient circumstances. In addition, Pseudomonas aeruginosa, which was the main source of infection in the patient, was a strain corresponding to the species selectivity of NPWT, which may have helped in wound healing [14]. Therefore, strains that cause infection are also to be considered when applying NPWT to infective lesions. This study highlights the significance of NPWT in wound healing in cases where bacterial excretion is insufficient.

Despite the advantages, NPWT may cause several complications (e.g., bleeding, infection, and pain) $[4,15]$. In general, NPWT is contraindicated in patients with osteomyelitis and other infectious lesions. In the present case, bone debridement and tunneling may have had an impact on the patient's clinical outcomes. However, it was difficult to conclude whether debridement alone was sufficient to treat the patient. Nevertheless, it appeared that the small bone tunnel promoted drainage, resulting in significant symptom improvement as well as osteomyelitis relief. Therefore, this study showed that NPWT should be considered in patients with osteomyelitis or complex wounds (e.g., uncontrolled and extensive infection in the dead space) to promote drainage and improve wound healing.

In complex wounds infected with MDR bacteria, NPWT may aid wound healing by reducing bacterial load. Despite 
recommendations against NPWT in patients with osteomyelitis, this study demonstrated that NPWT is an effective treatment option in selected patients with infected wounds, including osteomyelitis.

\section{Conflict of interest}

No potential conflict of interest relevant to this article was reported.

\section{ORCID iDs}

Taekeun Yoon https://orcid.org/0000-0002-1070-5165

Sang Wha Kim https://orcid.org/0000-0003-0430-3458

\section{References}

1. Argenta LC, Morykwas MJ. Vacuum-assisted closure: a new method for wound control and treatment. Clinical experience. Ann Plast Surg 1997;38:563-76.

2. Glass GE, Murphy G, Nanchahal J. Does negative-pressure wound therapy influence subjacent bacterial growth? A systematic review. J Plast Reconstr Aesthet Surg 2017;70: 1028-37.

3. Mendez-Eastman S. Guidelines for using negative pressure wound therapy. Adv Skin Wound Care 2001;14:314-22.

4. Gabriel A, Shores J, Bernstein B, et al. A clinical review of infected wound treatment with vacuum assisted closure (V.A.C.) therapy: experience and case series. Int Wound J 2009;6 Suppl 2(Suppl 2):1-25.

5. Orgill DP, Bayer LR. Negative pressure wound therapy: past, present and future. Int Wound J 2013;10 Suppl 1(Suppl 1):15-9.

6. Huang C, Leavitt T, Bayer LR, et al. Effect of negative pressure wound therapy on wound healing. Curr Probl Surg
2014;51:301-31.

7. Warriner R, Burrell R. Infection and the chronic wound: a focus on silver. Adv Skin Wound Care 2005;18 Suppl 1:212.

8. Glass GE, Murphy GF, Esmaeili A, et al. Systematic review of molecular mechanism of action of negative-pressure wound therapy. Br J Surg 2014;101:1627-36.

9. Liu D, Zhang L, Li T, et al. Negative-pressure wound therapy enhances local inflammatory responses in acute infected soft-tissue wound. Cell Biochem Biophys 2014;70:53947.

10. Weed T, Ratliff C, Drake DB. Quantifying bacterial bioburden during negative pressure wound therapy: does the wound VAC enhance bacterial clearance? Ann Plast Surg 2004;52:276-9.

11. Yusuf E, Jordan X, Clauss M, et al. High bacterial load in negative pressure wound therapy (NPWT) foams used in the treatment of chronic wounds. Wound Repair Regen 2013;21:677-81.

12. Acosta S, Bjorck M, Wanhainen A. Negative-pressure wound therapy for prevention and treatment of surgicalsite infections after vascular surgery. Br J Surg 2017;104: e75-84.

13. Monsen C, Wann-Hansson C, Wictorsson C, et al. Vacuum-assisted wound closure versus alginate for the treatment of deep perivascular wound infections in the groin after vascular surgery. J Vasc Surg 2014;59:145-51.

14. Moues CM, Vos MC, van den Bemd GJ, et al. Bacterial load in relation to vacuum-assisted closure wound therapy: a prospective randomized trial. Wound Repair Regen 2004; 12:11-7.

15. Li Z, Yu A. Complications of negative pressure wound therapy: a mini review. Wound Repair Regen 2014;22:45761. 